\title{
Aplicación de la técnica de sedimentación espontánea en tubo en el diagnóstico de parásitos intestinales.
}

\section{$\underline{\text { Artículo Original }}$}

Giovanni Pajuelo-Camachoํㅗㄹ Daniel Luján-Roca ${ }^{1}$, Bertha Paredes-Pérez², Raúl Tello-Casanova ${ }^{3}$.

${ }^{1}$ Universidad Nacional Federico Villarreal, ${ }^{2}$ Laboratorio de Microbiología y Parasitología, Hospital de Emergencias Pediátricas, ${ }^{3}$ Universidad Peruana Cayetano Heredia. Lima, Perú.

\section{RESUMEN.}

Objetivo. Describir un nuevo método de concentración simple, de bajo costo y de alta sensibilidad en el diagnóstico de parásitos intestinales.

Material y métodos. La "técnica de sedimentación espontánea en tubo" fue aplicada a 108 muestras fecales en pacientes del Hospital de Emergencias Pediátricas, Perú.

Resultados. La sedimentación espontánea mostró un mayor rendimiento (50.9\%) en comparación con el exámen directo (23.2\%) y la técnica de flotación con sulfato de zinc (25.9\%) y fue más eficiente en la detección de quistes de protozoos y huevos de helmintos intestinales.

Conclusiones. La técnica de sedimentación espontánea en tubo confirmó ser un método de concentración de alto rendimiento, y se convierte en una alternativa aplicable en países en desarrollo.

(Rev Biomed 2006; 17:96-101)

Palabras clave: parásitos intestinales, técnica de sedimentación espontánea en tubo, diagnóstico parasitológico.

\section{SUMMARY.}

Application of spontaneous tube sedimentation technique to diagnose intestinal parasites.

Objective. To describe a new low cost, highly sensitive method of simple concentration in the diagnosis of intestinal parasites.

Materials and methods. The technique of spontaneous sedimentation in tube was applied to 108 fecal samples in patients at the Hospital of Pediatric Emergencies, Perú.

Results. Spontaneous sedimentation showed a higher yield (50.9\%) in comparison with the direct exam (23.2\%) or the flotation technique with zinc sulphatel (25.9\%) and it was more efficient in the detection of cysts of protozoa and eggs of intestinal helminths.

Conclusions. The technique of spontaneous sedimentation in tube is confirmed to be a high yield method of concentration, and becomes an applicable alternative in developing countries.

(Rev Biomed 2006; 17:96-101)

Key words: intestinal parasites, technique of

Solicitud de sobretiros: Daniel Luján-Roca, Av. Garcilazo de la Vega 334, Depto. 301, Urb. La Colonial, Callao, Lima, Perú. Correo electrónico:d_lujan@starmedia.com 


\section{G Pajuelo-Camacho, D Luján-Roca, B Paredes-Pérez, R Tello-Casanova.}

spontaneous sedimentation in tube, parasitology diagnosis.

\section{INTRODUCCIÓN.}

Las infecciones parasitarias, producidas por protozoos y helmintos intestinales, afecta a más de dos billones de la población mundial (1) y constituye un problema de salud pública, especialmente en países en desarrollo con inadecuadas condiciones sanitarias (2).

El diagnóstico de las infecciones parasitarias intestinales se basa ampliamente en el análisis microscópico de las muestras fecales, que incluyen montajes húmedos directos, concentrados y frotis con tinción permanente. La cantidad de formas parasitarias en muestras de materias fecal, a menudo, es muy escasa y muy difíciles de detectar en preparados directos en fresco o en frotis teñidos; por lo tanto, siempre deben realizarse procedimientos de concentración (3).

En general, las dos técnicas de concentración utilizados con mayor frecuencia son los de sedimentación y de flotación $(3,4)$. El método de concentración por sedimentación con formalinaéter es el procedimiento más utilizado para concentrar quistes de protozoos, huevos y larvas de helmintos, y es más eficiente que los métodos de flotación (4). No obstante, debe tenerse cuidado durante el proceso de decantar el sobrenadante ya que pueden perderse una cantidad significativa de formas parasitarias. Además, el éter es un solvente orgánico peligroso y muy inflamable $(3,4)$. Por otro lado, el método de flotación con sulfato de zinc es útil para recuperar quistes de protozoos y huevos de helmintos; sin embargo, los huevos operculados, al igual que los de Schistosoma y los infértiles de Ascaris, no se obtienen con facilidad con este método $(3,4)$.

En los países en desarrollo, el alto costo y suministro controlado e irregular del éter y la carencia de centrífuga impide que muchos laboratorios utilizen esta técnica y otros procedimientos. En ese sentido, el énfasis en la búsqueda e importancia de desarrollar y utilizar técnicas sencillas, económicas y más eficaces, resulta considerable. Una prometedora técnica de concentración por sedimentación ha sido descrita (5). Evaluaciones preliminares de la técnica, muestran un mayor rendimiento en comparación con otras técnicas convencionales (6-10). Este estudio reporta la aplicación y utilización de la "técnica de sedimentación espontánea en tubo" (TSET) en el diagnóstico de parásitos intestinales.

\section{MATERIAL Y METODOS.}

Las muestras fecales de 108 niños con diagnóstico presuntivo de parasitosis intestinal, $y$ atendidos por consultorios externos u hospitalización del Hospital de Emergencias Pediátricas Lima-Perú, fueron colectadas para investigación. Una muestra de cada paciente pediátrico, evacuadas espontáneamente el mismo día, libres de orina y en cantidad necesaria fue examinada inmediatamente mediante la TSET, además del examen microscópico directo con solución salina y lugol y el método de flotación con sulfato de zinc (4). Se excluyeron del estudio, las muestras contaminadas con orina, insuficientes y de pacientes que estuvieron bajo tratamiento antiparasitario.

La TSET fue adaptada por el Dr. Raúl Tello (5) del Instituto de Medicina Tropical Alexander von Humboldt, Lima-Perú, y se llevó a cabo de acuerdo con las adaptaciones realizadas por el mismo autor $(6,10,11)$. Se separó aproximadamente 2-5 g de materia fecal y se homogenizó en 10 $\mathrm{mL}$ de solución salina hasta que se logró una suspensión adecuada. La mezcla fue vertida en un tubo cónico de plástico de $13 \times 2.5 \mathrm{~cm}$, de $50 \mathrm{~mL}$ de capacidad, filtrándola a través de gasa. Se completó el volumen final del tubo con más solución salina y se tapó herméticamente. Se agitó enérgicamente por 30 segundos y se dejó reposar por 45 minutos. Se eliminó el sobrenadante y se tomó con una pipeta una muestra del fondo el tubo. Se colocaron 3-4 gotas en dos láminas portaobjetos diferentes, agregándole luego gotas

\section{Revista Biomédica}


Técnica de sedimentación espontánea para diagnóstico de enteroparásitos.

de lugol a una de ellas. Finalmente, las láminas portaobjetos fueron cubiertas con laminillas de celofán de 6 × $2 \mathrm{~cm}$, y se observó al microscopio (100X y 400X).

\section{Análisis estadístico.}

Los resultados fueron analizados en Epi Info (versión 6.04 b). Luego se aplicó el test de Chi-cuadrado con corrección de Yates para la comparación estadística entre los métodos parasitológicos. Un valor de $\mathrm{p}<0.05$ fue considerado como el nivel crítico de significancia.

\section{RESULTADOS.}

Del total de 108 muestras fecales examinadas por los tres métodos parasitológicos, la TSET mostró un mayor rendimiento (50.9\%) en comparación con el exámen directo con solución salina y lugol (23.2.\%) y la técnica de flotación con sulfato de zinc (25.9\%) como se muestra en el cuadro 1 . Se encontró diferencia estadística significativa entre los métodos de diagnóstico $\left(\mathrm{x}^{2}=21.32 ; \mathrm{p}<0.001\right)$.

La TSET presentó mayor efectividad tanto en el diagnóstico de protozoos como de helmintos en relación con el exámen directo y el método de flotación con sulfato de zinc (cuadro 2).

La recuperación de las formas vacuoladas de Blastocystis hominis fue mayor con el procedimiento de sedimentación espontánea en relación al de flotación, principalmente. Además, la morfología de B. hominis fue mejor conservada con la TSET que con la técnica de flotación.

De las cuatro muestras que contenian ooquistes de Cyclospora cayetanensis, dos fueron diagnosticadas solamente por la sedimentación espontánea.

Por otro lado, la TSET fue el método más eficaz en el diagnóstico de helmintos, detectando el $100 \%$ de los casos. Dos muestras de heces positivas para huevos de Trichuris trichiura y Fasciola hepatica fueron también detectadas sólo por la técnica de sedimentación espontánea.

\section{DISCUSIÓN.}

La TSET obtuvo una mayor rendimiento en el diagnóstico de parásitos intestinales en comparación al exámen directo y la técnica de flotación con sulfato de zinc, utilizados como métodos de rutina en el Laboratorio de Microbiología y Parasitología del Hospital de Emergencias Pediátricas, LimaPerú. Estos hallazgos, confirman la eficiencia del procedimiento de sedimentación espontánea sobre estas y otras técnicas convencionales tales como el método de Ritchie, reportados en diversos estudios realizados Perú (6-10). En la TSET se utiliza una mayor cantidad de muestra (2-5 g) lo que explicaría su mayor rendimiento en contraste con los otros métodos utilizados. Además, permite la sedimentación espontánea de

\section{Cuadro 1}

\section{Rendimiento comparativo de la técnica de sedimentación espontánea en tubo en el} diagnóstico de parásitos intestinales.

\begin{tabular}{lcc}
\hline \multicolumn{1}{c}{ Método } & $\begin{array}{c}\text { Número de muestras } \\
\text { examinadas }\end{array}$ & $\begin{array}{c}\text { Positivos } \\
\mathrm{n}(\%)\end{array}$ \\
\hline Examen directo & 108 & $26(23.2)$ \\
Método de flotación $\left(\mathrm{ZnSO}_{4}\right)$ & 108 & $28(25.9)$ \\
TSET & 108 & $55(50.9)$ \\
\hline
\end{tabular}

TSET: Técnica de sedimentación espontánea en tubo $\left(x^{2}=21,32 ; p<0.001\right)$ 


\section{G Pajuelo-Camacho, D Luján-Roca, B Paredes-Pérez, R Tello-Casanova.}

\section{Cuadro 2}

Efectividad de los procedimientos: técnica de sedimentación espontánea en tubo, flotación con sulfato de zinc y examen directo en la detección de parásitos intestinales.

\begin{tabular}{|c|c|c|c|c|c|c|c|c|}
\hline \multirow{2}{*}{ Parásito } & & \multicolumn{2}{|c|}{ TSET } & \multicolumn{2}{|c|}{$\mathrm{ZnSO}_{4}$} & \multicolumn{2}{|c|}{$\overline{\text { DIRECTO }}$} & \multirow[t]{2}{*}{ TOTAL } \\
\hline & & $\mathrm{F}$ & $\%$ & $\%$ & $\mathrm{~F}$ & $\%$ & & \\
\hline Protozoos & & & & & & & & \\
\hline$\overline{\text { Blastocystis hominis }}$ & 37 & 100.0 & 3 & 8.1 & 7 & 18.9 & 37 & \\
\hline Entamoeba coli & & 10 & 90.9 & 8 & 72.7 & 3 & 27.3 & 11 \\
\hline Endolimax nana & & 9 & 81.8 & 4 & 36.4 & 6 & 54.5 & 11 \\
\hline Giardia duodenalis & & 11 & 100.0 & 10 & 90.9 & 10 & 90.9 & 11 \\
\hline Chilomastix mesnili & & 4 & 100.0 & 0 & 0 & 4 & 100.0 & 4 \\
\hline Cyclospora cayetanensis & 4 & 100.0 & 2 & 50.0 & 2 & 50.0 & 4 & \\
\hline $\begin{array}{l}\text { Iodamoeba butschlii } \\
\text { Helmintos }\end{array}$ & 3 & 100.0 & 1 & 33.3 & 2 & 66.7 & 3 & \\
\hline Ascaris lumbricoides & 7 & 100.0 & 4 & 57.1 & 1 & 14.3 & 7 & \\
\hline Hymenolepis nana & 5 & 100.0 & 3 & 60.0 & 2 & 40.0 & 5 & \\
\hline Enterobius vermicularis & 4 & 100.0 & 3 & 75.0 & 1 & 25.0 & 4 & \\
\hline Uncinaria & 3 & 100.0 & 2 & 66.7 & 0 & 0 & 3 & \\
\hline Trichuris trichiura & & 1 & 100.0 & 0 & 0 & 0 & 0 & 1 \\
\hline Fasciola hepatica & 1 & 100.0 & 0 & 0 & 0 & 0 & 1 & \\
\hline
\end{tabular}

TSET: Técnica de sedimentación espontánea en tubo

$\mathrm{ZnSO}_{4}$ : Método de flotación con sulfato de zinc

los diferentes parásitos, evitando que se pierdan algunos durante el proceso de centrifugación. Asimismo, la principal ventaja de esta técnica es que los organismos permanecen viables y no hay distorsión de las formas parasitarias.

Blastocystis hominis, fue identificado microscópicamente por la presencia de la forma vacuolar o de "cuerpo central" en el presente estudio. En general, los métodos de concentración son inadecuados para el diagnóstico de $B$. hominis debido a que estos causan distorsión de la forma vacuolar, multivacuolar y granular del organismo (12). No obstante, se ha sugerido que la sedimentación espontánea es un método apropiado para la separación de $B$. hominis de materia fecal (13) lo cual ha sido confirmado por nuestro estudio y otra investigación realizada en Perú (10). La baja sensibilidad de la técnica de flotación con sulfato de zinc en relación con la TSET podría deberse a la hipertonicidad del medio que ocasionaría la lisis de la forma vacuolar del parásito.

Revista Biomédica
La TSET demuestra ser un método efectivo en el hallazgo de ooquistes de Cyclospora cayetanensis, lo cual permitió maximizar su detección ya que la excreción de estos organismos es relativamente bajo, incluso en pacientes sintomáticos no inmunes (14). En efecto, la TSET se convierte en un método de concentración alternativo a las técnicas de flotación de Sheather y de sedimentación de étil acetato-formalina para el diagnóstico de este protozoo coccidio emergente, y actualmente utilizadas.

Cabe resaltar que la TSET viene siendo aplicada en el Instituto de Medicina Tropical Alexander von Humboldt de la Universidad Peruana Cayetano Heredia, así como en estudios de prevalencia de parasitosis intestinal en diferentes comunidades urbanas y rurales de Perú, con una sensibilidad mayor que las técnicas convencionales (15-20). Sin embargo, su adaptación en los hospitales y centros de salud de nuestro país aún no se ha llevado a cabo. 


\section{Técnica de sedimentación espontánea para diagnóstico de enteroparásitos.}

En conclusión, nuestros resultados confirman el alto rendimiento de la TSET en el diagnóstico tanto de protozoos como de helmintos, y debido a su simplicidad técnica, bajo costo y alta sensibilidad se convierte en una alternativa aplicable en países en desarrollo, principalmente en los centros de salud.

\section{AGRADECIMIENTOS.}

Un especial agradecimiento al Dr. Máximo Camiña (Hospital de Emergencias Pediátricas) por las facilidades brindadas para este estudio, al Lic. Ronald Torres (Hospital San Bartolomé) por el análisis estadístico.

\section{REFERENCIAS.}

1.- Morrone F, Carneiro J, Reis C, Cardozo C, Ubal C, de Carli G. Study of enteroparasites infection frecuency and chemotherapeutic agents used in pediatric patients in a community living in Porto Alegre, RS, Brazil. Rev Inst Med Trop S Paulo 2004; 46: 77-80.

2.- Savioli L, Bundy D, Tomkins A. Intestinal parasitic infection: a soluble public health problem. Trans R Soc Trop Med Hyg 1992; 86: 353-4.

3.- Koneman E, Allen S, Janda W, Scheckenberger P, Winn W. Diagnóstico Microbiológico. 5a. Edición, México: Ed. Médica Panamericana; 1999.

4.- Leventhal R, Cheadle R. Parasitología Médica. 3a. Edición, México: Ed. Interamericana, Mc Graw-Hill; 1992.

5.- Tello R. Empleo de una nueva técnica parasitológica rápida de sedimentación espontánea en el diagnóstico de protozoarios y helmintos. Resumen. V Jornadas Científicas. II Jornadas Científicas Estudiantiles. Lima-Perú. 1988, I164.

6.- Larragán M. Comparación de los principales métodos de diagnóstico para enteroparásitos. Tesis para optar el título de Médico Cirujano. Lima, Perú: Universidad Peruana Cayetano Heredia,1993.

7.- Vera L, Tello R, Terashima A, Alvarez H. Evaluación en campo de la técnica de sedimentación espontánea para el diagnóstico de enteroparasitosis. IX Jornadas Científicas “Carlos Monge Casinelli”. Rev Med Hered 1996; 7(Suppl.1): 50.
8.- Salas R, Rodríguez J. Evaluación de la técnica de sedimentación espontánea en el diagnóstico de enteroparásitos. III Congreso Peruano de Parasitología, 9, 1997.

9.- Maco V, Marcos L, Terashima A, Tello R, Samalvides F, Gotuzzo E. Técnica de Sedimentación Espontánea en Tubo (TSET): alto rendimiento en el diagnóstico de parásitos intestinales en comparación con otras técnicas coproparasitológicas. Rev Med Hered 2001;12 (Suppl.1): 111.

10.- Alvino A. Estudio parasitológico en la comunidad de Huayopampa (Huaral) y comparación de la Técnica de Sedimentación Espontánea en Tubo (TSET) con los Métodos Faust y Directo. Tesis para optar el título de Médico Cirujano. Lima, Perú: Universidad Peruana Cayetano Heredia, 2001.

11.- Tello R, Canales M. Técnicas de diagnóstico de enfermedades causadas por enteroparásitos. Diagnóstico 2000; 39: 197-8.

12. Stenzel D, Boreham P. Blastocystis hominis revisited. Clin Microbiol Rev 1996; 9: 563-84.

13.- Guimaraes S, Sogayar M. Blastocystis hominis: ocurrence in children and staff members of municipal daycare centers from Botucatu, Sao Paulo State, Brazil. Mem Inst Oswaldo Cruz 1993; 88: 427-9.

14.- Herwaldt B. Cyclospora cayetanensis: A Review, Focusing on the Outbreaks of Cyclosporiasis in the 1990s. Clin Infect Dis 2000; 31:1040-57.

15.- Marcos L, Maco V, Machicado A, Samalvides F, Terashima A, Quijano C. Diferencias de prevalencia de parasitosis intestinal entre los Hospitales Militar y Regional de Iquitos, Loreto-Perú. Diagnóstico 2002; 41: 185-7.

16.- Maco V, Raymundo L. Terashima A, Samalvides F, Miranda E, Espinoza J, et al. Fas2-ELISA y la técnica de sedimentación rápida modificada por Lumbreras en el diagnóstico de la infección por Fasciola hepatica. Rev Med Hered 2002; 13: 49-57.

17.- Marcos L, Maco V, Terashima A, Samalvides F, Gotuzzo E. Prevalencia de parasitosis intestinal en niños del valle del Mantaro, Jauja, Perú. Rev Med Hered 2002; 13: 85-9.

18.- Maco V, Marcos L, Terashima A, Samalvides F, Gotuzzo E. Distribución de la Enteroparasitosis en el Altiplano Peruano: Estudio en 6 comunidades rurales del departamento de Puno, Perú. Rev Gastroent Perú 2002; 22: 304-9. 
G Pajuelo-Camacho, D Luján-Roca, B Paredes-Pérez, R Tello-Casanova.

19. Marcos L, Maco V, Terashima A, Samalvides F, Miranda E, Gotuzzo E. Parasitosis intestinal en poblaciones urbana y rural en Sandia, Departamento de Puno, Perú. Parasitol Latinoam 2003; 58: 35-40.

20.- Marcos L, Maco V, Terashima A, Samalvides F, Miranda E, Tantalean M, y col. Hiperendemicidad de Fasciolosis humana en el Valle del Mantaro, Perú: Factores de riesgo de la infección por Fasciola hepatica. Rev Gastroent Perú 2004; 24: 158-64. 